TRANSACTIONS OF THE

AMERICAN MATHEMATICAL SOCIETY

Volume 349, Number 9, September 1997, Pages 3823-3836

S 0002-9947(97)01808-4

\title{
ON FROBENIUS ALGEBRAS AND THE QUANTUM YANG-BAXTER EQUATION
}

\author{
K. I. BEIDAR, Y. FONG, AND A. STOLIN
}

\begin{abstract}
It is shown that every Frobenius algebra over a commutative ring determines a class of solutions of the quantum Yang-Baxter equation, which forms a subbimodule of its tensor square. Moreover, this subbimodule is free of rank one as a left (right) submodule. An explicit form of a generator is given in terms of the Frobenius homomorphism. It turns out that the generator is invertible in the tensor square if and only if the algebra is Azumaya.
\end{abstract}

\section{InTRODUCTION}

In this paper we show that for any associative Frobenius $K$-algebra $A$, there exists a class of solutions of the quantum Yang-Baxter equation (QYBE). This class is a subbimodule of $A \otimes_{K} A$ under the operations $a \cdot(x \otimes y) \cdot b=a x \otimes y b$, $a, b, x, y \in A$, which is free of rank one with generator $Q$ as left (right) $A$-submodule. If $A$ is a free finitely generated $K$-module, then the property of $A$ to be a Frobenius algebra is equivalent to the existence of such an element $Q$. Finally we show that $Q$ is invertible if and only if $A$ is an Azumaya algebra. The paper is a continuation of [1] and it is inspired by Drinfel'd's article [4], where cases of quasi Frobenius and Frobenius Lie algebras over fields are considered. A connection between solutions of the classical Yang-Baxter equation (CYBE) and quasi Frobenius and Frobenius Lie algebras over fields was studied by Drinfel'd [4] and Stolin [12] and [13]. As far as we know no results about a connection between Frobenius algebras and solutions of QYBE have been obtained.

It is known that every Hopf algebra over a commutative ring $K$ with trivial Picard group, which is a finitely generated projective $K$-module, is a Frobenius algebra (see [10] and [8]). It turns out that this element $Q$ plays an important role in the structure theory of Hopf algebras over commutative rings (see [1]). We shall discuss the Hopf algebra case in a forthcoming paper.

In what follows $K$ is an associative commutative ring with 1 and $A$ an associative $K$-algebra with 1 . Given any $K$-module $M$ and $R \in \operatorname{End}\left(M \otimes_{K} M\right)$, we shall say that $R$ is a solution of the quantum Yang-Baxter equation (QYBE), if

$$
R^{12} R^{13} R^{23}=R^{23} R^{13} R^{12}
$$

Received by the editors August 4, 1995 and, in revised form, March 7, 1996.

1991 Mathematics Subject Classification. Primary 81R50, Secondary 16L60.

Key words and phrases. Quantum Yang-Baxter equation, Frobenius algebra, Azumaya algebra.

We would like to express our gratitude to the Swedish Academy of Science for the support of the visit of the first author to Sweden, during which this paper was finished. We are thankful to Professor B. Pareigis for fruitful discussions. 
where $R^{12}=R \otimes \mathrm{id}, R^{23}=\mathrm{id} \otimes R$, etc., in the standard way as maps $M \otimes_{K}$ $M \otimes_{K} M \rightarrow M \otimes_{K} M \otimes_{K} M$. The reader is referred to [5], [7] and [9] for a general discussion of QYBE.

Let $R=\sum_{i=1}^{n} a_{i} \otimes b_{i} \in A \otimes_{K} A$. Then $R$ acts on $A \otimes_{K} A$ via left multiplication and

$$
\begin{aligned}
& R^{12}=\sum_{i=1}^{n} a_{i} \otimes b_{i} \otimes 1, \quad R^{13}=\sum_{i=1}^{n} a_{i} \otimes 1 \otimes b_{i} \\
& R^{23}=\sum_{i=1}^{n} 1 \otimes a_{i} \otimes b_{i} \in A \otimes_{K} A \otimes_{K} A .
\end{aligned}
$$

Now let $L$ be a Lie algebra over $K$. An element $R=\sum_{i=1}^{n} a_{i} \otimes b_{i} \in L \otimes_{K} L$ is called a solution of CYBE, if

$$
\begin{gathered}
{\left[R^{12}, R^{13}\right]+\left[R^{12}, R^{23}\right]+\left[R^{13}, R^{23}\right]=0,} \\
\sum_{i=1}^{n} a_{i} \otimes b_{i}=-\sum_{i=1}^{n} b_{i} \otimes a_{i},
\end{gathered}
$$

where

$$
\begin{aligned}
& {\left[R^{12}, R^{13}\right]=\sum_{i, j=1}^{n}\left[a_{i}, a_{j}\right] \otimes b_{i} \otimes b_{j}, \quad\left[R^{12}, R^{23}\right]=\sum_{i, j=1}^{n} a_{i} \otimes\left[b_{i}, a_{j}\right] \otimes b_{j},} \\
& {\left[R^{13}, R^{23}\right]=\sum_{i, j=1}^{n} a_{i} \otimes a_{j} \otimes\left[b_{i}, b_{j}\right] \in L \otimes_{K} L \otimes_{K} L .}
\end{aligned}
$$

The reader is referred to [5], [12] and [13] for general discussion of CYBE.

Let $M$ be a $K$-module. We set $M^{*}=\operatorname{Hom}_{K}(M, K)$ and we shall write $\langle f, x\rangle$ for $f(x)$, where $f \in M^{*}, x \in M$. A bilinear form $B: M \times M \rightarrow K$ is called surgenerate if the mapping $\psi=\psi_{B}: M \rightarrow M^{*}$ given by the rule $\langle\psi(x), y\rangle=B(y, x)$ for all $x, y \in M$ is an isomorphism of $K$-modules (see [8, Lemma 2]).

Recall that a Lie algebra $L$ over $K$ is said to be quasi Frobenius if $L$ is a finitely generated projective $K$-module having a skew-symmetric surgenerate bilinear form $B: L \times L \rightarrow K$ such that

$$
B([x, y], z)+B([y, z], x)+B([z, x], y)=0 \quad \text { for all } x, y, z \in L .
$$

A Lie algebra $L$ over $K$ is called Frobenius if $L$ is a finitely generated projective $K$-module and there exists $\phi \in L^{*}$ such that the bilinear form $B_{\phi}(x, y)=\phi([x, y])$, $x, y \in L$, is surgenerate. The mapping $\phi$ is called a Frobenius homomorphism of $L$. Analogously an associative $K$-algebra $A$ is said to be Frobenius if $A$ is a finitely generated projective $K$-module and there exists $\phi \in A^{*}$ such that the bilinear form $B_{\phi}(x, y)=\phi(x y), x, y \in A$, is surgenerate. Further an algebra $A$ is called symmetric if $A$ is Frobenius and $\phi(x y)=\phi(y x)$ for all $x, y \in A$, where $\phi$ is a Frobenius homomorphism of $A$ (see [6]). Given a Frobenius (associative or Lie) algebra $B$ with Frobenius homomorphism $\phi$, we set $\psi=\psi_{B_{\phi}}$.

Let $A$ be a Frobenius algebra over $K$ with Frobenius homomorphism $\phi$. Since $A$ is a finitely generated projective $K$-module, there exist elements $e_{1}, e_{2}, \ldots, e_{n} \in A$ 
and $f^{1}, f^{2}, \ldots, f^{n} \in A^{*}$ such that

$$
x=\sum_{i=1}^{n} f^{i}(x) e_{i}=\sum_{i=1}^{n}\left\langle f^{i}, x\right\rangle e_{i}
$$

for all $x \in A$. In what follows we shall simply write $x=f^{i}(x) e_{i}=\left\langle f^{i}, x\right\rangle e_{i}$. Setting $e^{i}=\psi^{-1}\left(f^{i}\right), i=1,2, \ldots, n$, we note that $f^{i}(x)=\phi\left(x e^{i}\right)$ for all $x \in A, i=$ $1,2, \ldots, n$, and so $x=\phi\left(x e^{i}\right) e_{i}$ for all $x \in A$. We shall refer to $e_{1}, e_{2}, \ldots, e_{n}, e^{1}, e^{2}$, $\ldots, e^{n}$ and to $e_{1}, e_{2}, \ldots, e_{n}, f^{1}, f^{2}, \ldots, f^{n}$ as dual bases of $A$. Given any $x \in A$, there exists a unique element $x^{\prime} \in A$ such that $\phi\left(y x^{\prime}\right)=\left\langle\psi\left(x^{\prime}\right), y\right\rangle=\phi(x y)$ for all $y \in A$. One can easily check that the mapping $\alpha: A \rightarrow A$ given by the rule $\alpha(x)=x^{\prime}, x \in A$, is an automorphism of the algebra $A$. The automorphism $\alpha$ is called the Nakayama's automorphism of $A$.

The following result, due to Drinfel'd [4], is a starting point of the present paper.

Theorem 1.1. Let $L$ be a Lie algebra over a field $F$ with basis $e_{1}, e_{2}, \ldots, e_{n}$ and $\left(r^{i j}\right)_{j, j=1}^{n}$ a skew-symmetric nondegenerate $(n \times n)$-matrix over $F$ with inverse matrix $\left(b_{i j}\right)_{i, j=1}^{n}$. Denote by $B$ the bilinear form on $L$ with matrix $\left(b_{i j}\right)_{i, j=1}^{n}$ with respect to the basis $e_{1}, e_{2}, \ldots, e_{n}$. Then $R=r^{i j} e_{i} \otimes e_{j}$ is a solution of $C Y B E$ (1.2) and (1.3) if, and only if, $B$ satisfies (1.4) (i.e., $L$ is a quasi Frobenius Lie algebra).

\section{Preliminary Results}

Lemma 2.1. Let $M$ be a finitely generated projective $K$-module. Define the homomorphism of $K$-modules $\rho_{M}: M \otimes_{K} M \rightarrow \operatorname{Hom}_{K}\left(M^{*}, M\right)$ by the rule

$$
\rho_{M}(a \otimes b)(f)=f(a) b \quad \text { for all } a, b \in M
$$

and its consequences. Then $\rho_{M}$ is an isomorphism.

Proof. Clearly $\rho_{M}$ is an isomorphism if $M$ is free. Using localizations with respect to prime ideals of $K$, we complete the proof.

Proposition 2.2. Let $M$ be a finitely generated projective $K$-module with surgenerate bilinear form $B: M \times M \rightarrow M$ and $\psi=\psi_{B}$. Further let $e_{i} \in M, f^{i} \in M^{*}$, $i=1,2, \ldots, n$, be dual bases of $M$, and $e^{i}=\psi^{-1}\left(f^{i}\right), i=1,2, \ldots, n$. We set $Q=$ $e_{i} \otimes e^{i} \in M \otimes_{K} M$ and define the homomorphism $\chi=\chi_{M}: M \otimes_{K} M \rightarrow \operatorname{End}_{K}(M)$ by the rule

$$
\chi(a \otimes b)(x)=\langle\psi(b), x\rangle a=B(x, b) a, \quad a, b \in M,
$$

and its consequences. Further we define the mapping $\operatorname{Tr}: \operatorname{End}_{K}(M) \rightarrow K$ as follows:

$$
\operatorname{Tr}(\lambda)=\left\langle f^{i}, \lambda\left(e_{i}\right)\right\rangle=B\left(\lambda\left(e_{i}\right), e^{i}\right), \quad \lambda \in \operatorname{End}_{K}(M) .
$$

Then:

(1) $e^{1}, e^{2}, \ldots, e^{n}$ generate the $K$-module $M$;

(2) $x=B\left(x, e^{i}\right) e_{i}=B\left(e_{i}, x\right) e^{i}$ for all $x \in M$;

(3) $\chi$ is an isomorphism of $K$-modules;

(4) $Q=\rho_{M}^{-1}\left(\psi^{-1}\right)=\chi^{-1}\left(\operatorname{id}_{M}\right)$;

(5) The element $Q$ does not depend on the choice of dual bases $e_{i}, e^{i}, i=1, \ldots, n$, of $M$;

(6) The mapping $T r$ does not depend on the choice of the dual bases $e_{i}, e^{i}, i=$ $1, \ldots, n$, of $M$; 
(7) If $M$ is a free $K$-module, then $T r$ is the trace function on $\operatorname{End}_{K}(M)$.

Proof. (1) Since $\psi$ is an isomorphism of $K$-modules $M$ and $M^{*}$, it is sufficient to show that $f^{1}, f^{2}, \ldots, f^{n}$ generate the $K$-module $M^{*}$. Recall that $x=f^{i}(x) e_{i}$ for all $x \in M$. Let $f \in M^{*}$. Then

$$
f(x)=f\left(f^{i}(x) e_{i}\right)=f^{i}(x) f\left(e_{i}\right)=\left\langle f\left(e_{i}\right) f^{i}, x\right\rangle,
$$

and so $f=f\left(e_{i}\right) f^{i}$, which proves (1).

(2) Since $f^{i}(x)=B\left(x, e^{i}\right), x=f^{i}(x) e_{i}=B\left(x, e^{i}\right) e_{i}$ for all $x \in M$. Given $x \in M$, we set $y=B\left(e_{i}, x\right) e^{i}$. Then

$$
B(z, y)=B\left(z, B\left(e_{i}, x\right) e^{i}\right)=B\left(e_{i}, x\right) B\left(z, e^{i}\right)=B\left(B\left(z, e^{i}\right) e_{i}, x\right)=B(z, x)
$$

for all $z \in M$. Since $B$ is surgenerate, $y=x$.

(3) Define a homomorphism $\tau: M \otimes_{K} M^{*} \rightarrow \operatorname{End}_{K}(M)$ by the rule

$$
\tau(a \otimes f)(x)=f(x) a, a \in M, f \in M^{*},
$$

and its consequences. It is well-known that $\tau$ is an isomorphism of $K$-modules. Since $\chi=\tau \circ(1 \otimes \psi), \chi$ is an isomorphism of $K$-modules.

(4) We have $\rho_{M}(Q)\left(f^{k}\right)=f^{k}\left(e_{i}\right) e^{i}=B\left(e_{i}, e^{k}\right) e^{i}=e^{k}$ for all $k=1,2, \ldots, n$ by (2). On the other hand $\psi^{-1}\left(f^{k}\right)=e^{k}$ for all $k=1,2, \ldots, n$. Since $f^{1}, f^{2}, \ldots, f^{n}$ generate the $K$-module $M^{*}, \psi^{-1}=\rho_{M}(Q)$, and so $Q=\rho_{M}^{-1}\left(\psi^{-1}\right)$. Further $\chi(Q)(x)=B\left(x, e^{i}\right) e_{i}=x$ for all $x \in M$ by (2). Therefore $\chi(Q)=\operatorname{id}_{M}$ and hence $Q=\chi^{-1}\left(\operatorname{id}_{M}\right)$.

(5) follows directly from (4).

(6) Given $\lambda \in \operatorname{End}_{K}(M)$, define the mapping $\Lambda: M \otimes_{K} M \rightarrow K$ by the rule $\Lambda(a \otimes b)=B(\lambda(a), b)$ and its consequences. Obviously $\Lambda$ is a well-defined homomorphism of $K$-modules and $\operatorname{Tr}(\lambda)=\Lambda(Q)$. It follows from (5) that $\operatorname{Tr}(\lambda)$ does not depend on the choice of the dual bases $e_{i}, e^{i}, i=1,2, \ldots, n$, of $M$.

(7) Suppose that $M$ is a free $K$-module with free basis $u_{1}, u_{2}, \ldots, u_{m}$. Clearly there exist $u^{1}, u^{2}, \ldots, u^{m} \in M$ such that $B\left(u_{i}, u^{j}\right)=\delta_{i j}$ for all $i, j=1,2, \ldots, m$. By (6) $\operatorname{Tr}(\lambda)=B\left(\lambda\left(u_{i}\right), u^{i}\right)$, and hence $\operatorname{Tr}(\lambda)$ is the trace of $\lambda$.

Lemma 2.3. Let $M$ be a finitely generated free $K$-module with surgenerate bilinear form $B: M \times M \rightarrow M$ and $\psi=\psi_{B}$. Further let $e_{1}, e_{2}, \ldots, e_{n} \in M$ be a free basis of $M$. Choose $e^{1}, e^{2}, \ldots, e^{n} \in M$ such that $B\left(e_{i}, e^{j}\right)=\delta_{i j}$ for all $i, j=1,2, \ldots, n$. We set $Q=e_{i} \otimes e^{i} \in M \otimes_{K} M$ and write $e^{i}=\sum_{j=1}^{n} r^{j i} e_{j}$, where $r^{i j} \in K, i=1,2, \ldots, n$. Next we set $b_{i j}=\phi\left(e_{i} e_{j}\right), B=\left(b_{i j}\right)_{i, j=1}^{n} \in M_{n}(K)$ and $R=\left(r^{j i}\right)_{i, j=1}^{n}$. Then:

(1) $R B=E=B R$, where $E$ is the identity of $M_{n}(K)$;

(2) $Q=\sum_{i, j=1}^{n} r^{j i} e_{i} \otimes e_{j}$.

Proof. We see that

$$
\delta_{j i}=B\left(e_{j}, e^{i}\right)=B\left(e_{j}, \sum_{k=1}^{n} r^{k i} e_{k}\right)=\sum_{k=1}^{n} B\left(e_{j}, e_{k}\right) r^{k i}=\sum_{k=1}^{n} b_{j k} r^{k i},
$$

which proves (1). The last statement is obvious.

Here we close this section with following result (see [9, p. 15]).

Lemma 2.4. Let $A$ be an associative $K$-algebra and $Q \in A \otimes_{K} A$. Denote by $T$ the twist map $T: A \otimes_{K} A \rightarrow A \otimes_{K} A$ given by the rule $T(a \otimes b)=b \otimes a, a, b \in A$, and its consequences. Then the element $Q$ satisfies the relation $Q^{12} Q^{23} Q^{12}=Q^{23} Q^{12} Q^{23}$ if, and only if, the element $R=Q T \in \operatorname{End}_{K}\left(A \otimes_{K} A\right)$ is a solution of $Q Y B E$ (1.1). 


\section{Frobenius Algebras and YBE}

Theorem 3.1. Let $L$ be a Lie algebra over a commutative ring $K$ with surgenerate bilinear form $B: L \times L \rightarrow K$. Suppose that $L$ is a finitely generated projective $K$ module. Further let $e_{i}, e^{i}, i=1,2, \ldots, n$, be dual bases of $L$. We set $Q=e_{i} \otimes e^{i} \in$ $L \otimes_{K}$ L. Then:

(1) $Q=\rho_{L}^{-1}\left(\psi^{-1}\right)=\chi_{L}^{-1}\left(\operatorname{id}_{L}\right)$;

(2) The element $Q$ does not depend on the choice of dual bases $e_{i}, e^{i}, i=1, \ldots, n$, of $L$;

(3) The element $Q$ satisfies CYBE (1.2) and (1.3) if, and only if, $B$ is skewsymmetric and satisfies (1.4) (i.e., $L$ is quasi Frobenius).

Proof. The first two statements follows from Proposition 2.2 (4) and (5). Next suppose that $Q$ satisfies CYBE. Then from (1.3) we see that

$$
e^{j} \otimes e_{j}=T\left(e_{j} \otimes e^{j}\right)=-e_{j} \otimes e^{j},
$$

where $T$ is the twist map. We claim that $B$ is skew-symmetric. Indeed, let $m$ be any maximal ideal of $K$ and $\gamma: L \rightarrow L_{m}$ be the canonical homomorphism of the $K$-module $L$ into the $K_{m}$-module $L_{m}$, where $L_{m}$ is the localization of $L$ with respect to $m$. Clearly the bilinear form $B$ induces the surgenerate bilinear form $B_{m}: L_{m} \times L_{m} \rightarrow K_{m}$. It is enough to show that $B_{m}$ is skew-symmetric. Since $K_{m}$ is a local ring, $L_{m}$ is a free $K_{m}$-module. Let $u_{1}, u_{2}, \ldots, u_{t}$ be a basis of $L_{m}$ and $u_{i}, u^{i}, i=1,2, \ldots, t$, dual bases of $L_{m}$. Since $\gamma\left(e_{j}\right), \gamma\left(e^{j}\right), j=1,2, \ldots, n$, are dual bases of $L_{m}$ as well, it follows from Proposition $2.2(5)$ that $\gamma\left(e_{j}\right) \otimes \gamma\left(e^{j}\right)=u_{i} \otimes u^{i}$. In particular

$$
u^{i} \otimes u_{i}=T\left(u_{i} \otimes u^{i}\right)=-u_{i} \otimes u^{i} .
$$

Choosing $w^{i} \in L_{m}$ such that $B_{m}\left(u^{j}, w^{i}\right)=\delta_{i j}$ for all $1 \leq i, j \leq t$, we conclude from Proposition $2.2(5)$ that $u^{i} \otimes w^{i}=u_{i} \otimes u^{i}=-u^{i} \otimes u_{i}$ and so $w^{i}=-u_{i}$ for all $i=1,2, \ldots, t$. Therefore

$$
B_{m}\left(u_{i}, u^{j}\right)=\delta_{i j}=B_{m}\left(u^{j}, w^{i}\right)=-B_{m}\left(u^{j}, u_{i}\right) \quad \text { for all } 1 \leq i, j \leq t .
$$

Since both sets $u_{1}, u_{2}, \ldots, u_{t}$ and $u^{1}, u^{2}, \ldots, u^{t}$ generate the $K_{m}$-module $L_{m}$, it follows that $B_{m}$ is skew-symmetric, and so $B$ is skew-symmetric as well.

To complete the proof of the theorem it is enough to show that if $B_{m}$ is skewsymmetric, then the expression $\Theta=\left[Q^{12}, Q^{13}\right]+\left[Q^{12}, Q^{23}\right]+\left[Q^{13}, Q^{23}\right]$ equals zero if, and only if,

$$
B_{m} \quad \text { satisfies (1.4) }
$$

We have

$$
\begin{aligned}
{\left[Q^{12}, Q^{13}\right] } & =\left[u_{i}, u_{j}\right] \otimes u^{i} \otimes u^{j}=B_{m}\left(\left[u_{i}, u_{j}\right], u^{k}\right) u_{k} \otimes u^{i} \otimes u^{j} \\
{\left[Q^{12}, Q^{23}\right] } & =u_{k} \otimes\left[u^{k}, u_{j}\right] \otimes u^{j}=u_{k} \otimes B_{m}\left(u_{i},\left[u^{k}, u_{j}\right]\right) u^{i} \otimes u^{j} \\
& =B_{m}\left(u_{i},\left[u^{k}, u_{j}\right]\right) u_{k} \otimes u^{i} \otimes u^{j}=B_{m}\left(\left[u_{j}, u^{k}\right], u_{i}\right) u_{k} \otimes u^{i} \otimes u^{j} .
\end{aligned}
$$


Since $u_{i} \otimes u^{i}=-u^{i} \otimes u_{i}$,

$$
\begin{aligned}
{\left[Q^{13}, Q^{23}\right] } & =\left[Q^{13}, 1 \otimes u_{i} \otimes u^{i}\right]=-\left[Q^{13}, 1 \otimes u^{i} \otimes u_{i}\right] \\
& =u_{k} \otimes u_{i} \otimes\left[u^{k}, u^{i}\right]=-u_{k} \otimes u^{i} \otimes\left[u^{k}, u_{i}\right] \\
& =-u_{k} \otimes u^{i} \otimes B_{m}\left(u_{j},\left[u^{k}, u_{i}\right]\right) u^{j}=B_{m}\left(\left[u^{k}, u_{i}\right], u_{j}\right) u_{k} \otimes u^{i} \otimes u^{j} \\
\Theta & =\left(B_{m}\left(\left[u_{i}, u_{j}\right], u^{k}\right)+B_{m}\left(\left[u_{j}, u^{k}\right], u_{i}\right)+B_{m}\left(\left[u^{k}, u_{i}\right], u_{j}\right)\right) u_{k} \otimes u^{i} \otimes u^{j},
\end{aligned}
$$

and so $\Theta=0$ if, and only if,

$$
B_{m}\left(\left[u_{i}, u_{j}\right], u^{k}\right)+B_{m}\left(\left[u_{j}, u^{k}\right], u_{i}\right)+B_{m}\left(\left[u^{k}, u_{i}\right], u_{j}\right)=0
$$

for all $1 \leq i, j, k \leq n$. The latter is equivalent to (3.1), which completes the proof.

Here we note that if $L$ is a quasi Frobenius Lie algebra over a field $F$, then by Lemma 2.3, $r^{i j} e_{i} \otimes e_{j}=e^{j} \otimes e_{j}=-Q$, and so $-Q$ is the solution of CYBE from Theorem 1.1.

Given an algebra $A$, we set

$$
\mathrm{Q}(A)=\left\{Q \in A \otimes_{K} A \mid(1 \otimes a) Q=Q(a \otimes 1) \quad \text { for all } a \in A\right\} .
$$

We consider $A \otimes_{K} A$ as an $A$ - $A$-bimodule under the multiplications $a \cdot(x \otimes y)=a x \otimes y$ and $(x \otimes y) \cdot b=x \otimes y b$ for all $a, b, x, y \in A$.

Lemma 3.2. Let $A$ be a K-algebra and $Q=a_{i} \otimes b_{i} \in \mathrm{Q}(A)$. Then:

(1) $Q^{12} Q^{13}=Q^{23} Q^{12}=Q^{13} Q^{23}$;

(2) $Q^{12} Q^{23} Q^{12}=Q^{23} Q^{12} Q^{23}$;

(3) $\mathrm{Q}(A)$ is a subbimodule of $A \otimes_{K} A$.

Proof. (1) We have that

$$
\begin{aligned}
Q^{12} Q^{13} & =a_{i} a_{j} \otimes b^{i} \otimes b^{j}=\left[\left(a_{i} \otimes b^{i}\right)\left(a_{j} \otimes 1\right)\right] \otimes b^{j} \\
& =\left[\left(1 \otimes a_{j}\right)\left(a_{i} \otimes b^{i}\right)\right] \otimes b^{j}=a_{i} \otimes a_{j} b^{i} \otimes b^{j} \\
& =Q^{23} Q^{12} \\
& =a_{i} \otimes\left[\left(a_{j} \otimes b^{j}\right)\left(b^{i} \otimes 1\right)\right]=a_{i} \otimes\left[\left(1 \otimes b^{i}\right)\left(a_{j} \otimes b^{j}\right)\right]=a_{i} \otimes a_{j} \otimes b^{i} b^{j} \\
& =Q^{13} Q^{23} .
\end{aligned}
$$

From (1) we see that

$$
Q^{12} Q^{23} Q^{12}=Q^{12}\left(Q^{23} Q^{12}\right)=Q^{12} Q^{13} Q^{23}=\left(Q^{12} Q^{13}\right) Q^{23}=Q^{23} Q^{12} Q^{23} .
$$

The last statement is obvious. The proof is complete.

Recall that a $K$-algebra $A$ is called separable if it is a projective right $A \otimes_{K} A^{\circ}$ module, where $A^{\circ}$ is the opposite algebra to $A$ and $x \cdot(a \otimes b)=b x a$ for all $x, a, b \in$ $A$. According to [3, Proposition 1.1] $A$ is separable if and only if there exists an idempotent $e=\sum a_{i} \otimes b_{i} \in A \otimes_{K} A^{\circ}$ such that $\sum b_{i} a_{i}=1$ and $e *(a \otimes 1-1 \otimes a)=0$ for all $a \in A$. Here $*$ is the multiplication in $A \otimes_{K} A^{\circ}$. This element $e$ is called a separability idempotent for $A$. Considering $e$ as an element of $A \otimes_{K} A$ we conclude that $e(a \otimes 1)=(1 \otimes a) e$ for all $a \in A$, and therefore we have the following

Corollary 3.3. Let $A$ be a separable $K$-algebra with separability idempotent $Q$. Then $Q$ satisfies the conditions (1) and (2) of Lemma 3.2. 
Theorem 3.4. Let $A$ be a Frobenius algebra over a commutative ring $K$ with Nakayama's automorphism $\alpha$ and let $e_{i}, e^{i}, i=1,2, \ldots, n$, be dual bases of $A$. We set $Q=e_{i} \otimes e^{i} \in A \otimes_{K} A$ and denote by $T$ the twist map $T: A \otimes_{K} A \rightarrow A \otimes_{K} A$. Then:

(1) $Q=\rho_{A}^{-1}\left(\psi^{-1}\right)=\chi_{A}^{-1}\left(\operatorname{id}_{A}\right)$;

(2) The element $Q$ does not depend on the choice of dual bases $e_{i}, e^{i}, i=1, \ldots, n$, of $A$;

(3) $(a \otimes b) Q=Q(b \otimes \alpha(a))$ for all $a, b \in A$;

(4) $x e_{i} z e^{i}=e_{i} z e^{i} \alpha(x)$ and $x e^{i} y e_{i}=e^{i} y e_{i} x$ for all $x, z \in A$;

(5) $Q^{12} Q^{13}=Q^{23} Q^{12}=Q^{13} Q^{23}$;

(6) The element $Q$ satisfies the braid relation

$$
Q^{12} Q^{23} Q^{12}=Q^{23} Q^{12} Q^{23}
$$

(7) $R=Q T \in \operatorname{End}_{K}\left(A \otimes_{K} A\right)$ is a solution of $Q Y B E$ (1.1);

(8) $\mathrm{Q}(A)$ is a free rank one left (right) A-submodule of $A \otimes_{K} A$ with basis $\{Q\}$.

Proof. The first two statements follow directly from Proposition 2.2 (4) and (5).

(3) According to Proposition 2.2 (2) we have

$$
\begin{aligned}
(a \otimes b) Q & =a e_{i} \otimes b e^{i}=a e_{i} \otimes \phi\left(e_{j} b e^{i}\right) e^{j}=a \phi\left(e_{j} b e^{i}\right) e_{i} \otimes e^{j} \\
& =a e_{j} b \otimes e^{j}=\phi\left(a e_{j} e^{i}\right) e_{i} b \otimes e^{j}=\phi\left(e_{j} e^{i} \alpha(a)\right) e_{i} b \otimes e^{j} \\
& =e_{i} b \otimes \phi\left(e_{j} e^{i} \alpha(a)\right) e^{j}=e_{i} b \otimes e^{i} \alpha(a)=Q(b \otimes \alpha(a)) .
\end{aligned}
$$

(4) Consider $A \otimes_{K} A$ as an $A$ - $A$-bimodule under the operation

$$
x \cdot(a \otimes b) \cdot y=(x \otimes 1)(a \otimes b)(1 \otimes y)=x a \otimes b y,
$$

$x, y, a, b \in A$. Given $z \in A$, the mapping $\lambda_{z}: A \otimes A \rightarrow A$ given by the rule $\lambda_{z}(a \otimes b)=a z b$ is a homomorphism of bimodules. By (3) it follows that

$$
x e_{i} z e^{i}=\lambda_{z}\left([x \otimes 1]\left[e_{i} \otimes e^{i}\right]\right)=\lambda_{z}\left(\left[e_{i} \otimes e^{i}\right][1 \otimes \alpha(x)]\right)=e_{i} z e^{i} \alpha(x) .
$$

Defining $x *(a \otimes b) * y=a y \otimes x b$ and $\lambda_{z}(a \otimes b)=b z a$, one can prove the second equality.

Equalities (5) and (6) follow from Lemma 3.2. The statement (7) follows from (6) and Lemma 2.4.

(8) According to Lemma $3.2(3), \mathrm{Q}(A)$ is a subbimodule of $A \otimes_{K} A$. Since $A \cdot Q=Q \cdot \alpha(A)$, it is enough to show that $\mathrm{Q}(A)$ is a free right $A$-submodule of $A \otimes_{K} A$ with basis $Q$. Suppose that $Q \cdot a=0$ for some $a \in A$. Consider a homomorphism $\tau: A \otimes_{K} A \rightarrow A$ of right $A$-modules defined as follows: $\tau(x \otimes y)=\phi(x) y$ for all $x, y \in A$. Then

$$
0=\tau(Q \cdot a)=\tau(Q) a=\left(\sum_{i=1}^{n} \phi\left(e_{i}\right) e^{i}\right) a=a
$$

because $1=\sum_{i=1}^{n} \phi\left(1 \cdot e_{i}\right) e^{i}$. Therefore $a=0$, and it is enough to prove that $\mathrm{Q}(A)=Q \cdot A$. Localizations with respect to maximal ideals of $K$ reduce the problem to the case when $A$ is a free $K$-module. According to (2) we then may assume that $e_{1}, e_{2}, \ldots, e_{n}$ is a $K$-basis of $A$. Now choose any $Q^{\prime} \in \mathrm{Q}(A)$. We claim that $Q^{\prime}=Q \cdot a$ for some $a \in A$. Indeed, write $Q^{\prime}=\sum_{i=1}^{n} e_{i} \otimes b_{i}$, where 


$$
\begin{aligned}
& b_{1}, b_{2}, \ldots, b_{n} \in A \text {. Then } \\
& \begin{aligned}
\sum_{j=1}^{n} e_{j} \otimes x b_{j} & =(1 \otimes x) Q^{\prime}=Q^{\prime}(x \otimes 1)=\sum_{i=1}^{n} e_{i} x \otimes b_{i} \\
& =\sum_{i=1}^{n}\left(\sum_{j=1}^{n} \phi\left(e_{i} x e^{j}\right) e_{j}\right) \otimes b_{i}=\sum_{j=1}^{n} e_{j} \otimes\left(\sum_{i=1}^{n} \phi\left(e_{i} x e^{j}\right) b_{i}\right)
\end{aligned}
\end{aligned}
$$

and hence $x b_{j}=\sum_{i=1}^{n} \phi\left(e_{i} x e^{j}\right) b_{i}$ for all $j=1,2, \ldots, n, x \in A$. Define a homomorphism $\sigma: A \rightarrow A$ of $K$-modules by the rule $\sigma\left(e^{j}\right)=b_{j}$ for all $j=1,2, \ldots, n$. Then

$$
\sigma\left(x e^{j}\right)=\sigma\left(\sum_{i=1}^{n} \phi\left(e_{i} x e^{j}\right) e^{i}\right)=\sum_{i=1}^{n} \phi\left(e_{i} x e^{j}\right) \sigma\left(e^{i}\right)=\sum_{i=1}^{n} \phi\left(e_{i} x e^{j}\right) b_{i}=x b_{j}
$$

for all $j=1,2, \ldots, n$. Therefore $\sigma$ is a homomorphism of left $A$-modules, and so there exists an element $a \in A$ such that $\sigma(y)=y a$ for all $y \in A$. In particular $b_{j}=\sigma\left(e^{j}\right)=e^{j} a$ for all $j=1,2, \ldots, n$, and thus $Q^{\prime}=Q \cdot a$. The proof is complete.

The next theorem is a converse of Theorem 3.4.

Theorem 3.5. Let $A$ be a $K$-algebra which is a free $K$-module with two bases $e_{1}, e_{2}, \ldots, e_{n}$ and $e^{1}, e^{2}, \ldots, e^{n}$ and $Q=e_{i} \otimes e^{i} \in A \otimes_{K} A$. Then the following conditions are equivalent:

(1) $Q^{12} Q^{13}=Q^{23} Q^{12}$;

(2) $Q^{12} Q^{13}=Q^{13} Q^{23}$;

(3) $Q^{23} Q^{12}=Q^{13} Q^{23}$;

(4) $(1 \otimes a) Q=Q(a \otimes 1)$ for all $a \in A$;

(5) $A$ is a Frobenius algebra with Frobenius homomorphism $\phi: A \rightarrow K$ such that $\phi\left(e_{i} e^{j}\right)=\delta_{i j}$ for all $i, j=1,2, \ldots, n$.

Proof. First of all we shall prove that the conditions (1) - (4) are equivalent. In view of Lemma 3.2 it is enough to show that each of the conditions (1), (2), and (3) implies (4). We shall show that $(3) \Rightarrow(4)$, since the others can be proved analogously. From (3) it follows that

$$
Q^{23} Q^{12}=e_{i} \otimes e_{j} e^{i} \otimes e^{j}=e_{i} \otimes e_{j} \otimes e^{i} e^{j}=Q^{13} Q^{23}
$$

and so $\sum_{j} e_{j} e^{i} \otimes e^{j}=\sum_{j} e_{j} \otimes e^{i} e^{j}$ for all $i=1,2, \ldots, n$, i.e., $Q\left(e^{i} \otimes 1\right)=\left(1 \otimes e^{i}\right) Q$ for all $i$. Since $e^{i}, i=1,2, \ldots, n$, is a basis of the $K$-module $A$, the implication (3) $\Rightarrow(4)$ is proved and so all the conditions (1) - (4) are equivalent.

Taking into account Theorem 3.4, we have only to prove that (4) implies (5). To this end we define a bilinear map $B: A \times A \rightarrow K$ as follows: $B\left(e_{i}, e^{j}\right)=\delta_{i j}$ for all $i, j=1,2, \ldots, n$. Since $e_{1}, e_{2}, \ldots, e_{n}$ and $e^{1}, e^{2}, \ldots, e^{n}$ are two bases of the $K$-module $A$, it follows that $B$ is a well-defined surgenerate bilinear form. We claim that $B(x y, z)=B(x, y z)$ for all $x, y, z \in A$. Indeed, it is enough to show that $B\left(e_{j} y, e^{i}\right)=B\left(e_{j}, y e^{i}\right)$ for all $i, j=1,2, \ldots, n$ and $y \in A$. First of all we note that $a=B\left(e_{i}, a\right) e^{i}=B\left(a, e^{i}\right) e_{i}$ for all $a \in A$. From (4) we see that $e_{i} \otimes y e^{i}=e_{j} y \otimes e^{j}$ and hence

$$
e_{i} \otimes B\left(e_{j}, y e^{i}\right) e^{j}=B\left(e_{j} y, e^{i}\right) e_{i} \otimes e^{j}
$$


Thus $B\left(e_{j}, y e^{i}\right)=B\left(e_{j} y, e^{i}\right)$ for all $i, j=1,2, \ldots, n$ and $y \in A$. Setting $\phi(x)=$ $B(1, x)$, we conclude that $\phi$ is a Frobenius homomorphism and

$$
\phi\left(e_{i} e^{j}\right)=B\left(1, e_{i} e^{j}\right)=B\left(e_{i}, e^{j}\right)=\delta_{i j}
$$

for all $i, j=1,2, \ldots, n$. The proof is complete.

We recall that a Frobenius $K$-algebra $A$ with Frobenius homomorphism $\phi: A \rightarrow$ $K$ is called symmetric if $\phi(x y)=\phi(y x)$ for all $x, y \in A$. In this particular case the results obtained above for Frobenius algebras admit some refinements. We begin with the following lemma.

Lemma 3.6. Let $A$ be a $K$-algebra and $Q=e_{i} \otimes e^{i} \in A \otimes_{K} A$. Suppose that $(a \otimes b) Q=Q(b \otimes a)$. Then:

(1) $Q^{12} Q^{13}=Q^{23} Q^{12}=Q^{13} Q^{23}$ and $Q^{12} Q^{23}=Q^{23} Q^{13}=Q^{13} Q^{12}$;

(2) $Q^{12} Q^{13} Q^{23}=Q^{23} Q^{13} Q^{12}$.

Proof. The first statement of (1) is proved in Lemma 3.2 and the second can be proved similarly. Let us deduce (2). We have

$$
Q^{12} Q^{13} Q^{23}=\left(Q^{12} Q^{13}\right) Q^{23}=Q^{23} Q^{12} Q^{23}=Q^{23}\left(Q^{12} Q^{23}\right)=Q^{23} Q^{13} Q^{12} .
$$

The proof is complete.

Theorem 3.7. Let $A$ be a symmetric algebra over a commutative ring $K$ with Frobenius homomorphism $\phi$ and let $e_{i}, e^{i}, i=1,2, \ldots, n$, be dual bases of $A$. We set $Q=e_{i} \otimes e^{i} \in A \otimes_{K} A$ and denote by $T$ the twist map $T: A \otimes_{K} A \rightarrow A \otimes_{K} A$. Then:

(1) $(a \otimes b) Q=Q(b \otimes a)$ for all $a, b \in A$;

(2) $Q^{12} Q^{13}=Q^{23} Q^{12}=Q^{13} Q^{23}$ and $Q^{12} Q^{23}=Q^{23} Q^{13}=Q^{13} Q^{12}$;

(3) The element $Q$ is a solution of $Q Y B E$ (1.1), and $T Q=Q$.

Proof. Since $A$ is symmetric, the Frobenius homomorphism satisfies the condition $\phi(x y)=\phi(y x)$ for all $x, y \in A$. Therefore the corresponding Nakayama automorphism $\alpha$ is the identity mapping of $A$. It follows from Theorem 3.4 that

$$
(a \otimes b) Q=Q(b \otimes a) \quad \text { for all } a, b \in A .
$$

The last two statements follow immediately from Lemma 3.6.

The above theorem has a converse in the following sense.

Theorem 3.8. Let $A$ be a $K$-algebra which is a free $K$-module with two bases $e_{1}, e_{2}, \ldots, e_{n}$ and $e^{1}, e^{2}, \ldots, e^{n}$. Further let $Q=e_{i} \otimes e^{i} \in A \otimes_{K} A$. Then the following conditions are equivalent:

(1) $Q^{12} Q^{13}=Q^{23} Q^{12}$ and $Q^{12} Q^{23}=Q^{23} Q^{13}$;

(2) $Q^{12} Q^{13}=Q^{13} Q^{23}$ and $Q^{12} Q^{23}=Q^{13} Q^{12}$;

(3) $Q^{23} Q^{12}=Q^{13} Q^{23}$ and $Q^{23} Q^{13}=Q^{13} Q^{12}$;

(4) $(a \otimes b) Q=Q(b \otimes a)$ for all $a \in A$;

(5) $A$ is a symmetric algebra with Frobenius homomorphism $\phi: A \rightarrow K$ such that $\phi\left(e_{i} e^{j}\right)=\delta_{i j}$ for all $i, j=1,2, \ldots, n$.

Proof. We have already proved in Lemma 3.6 that the fourth condition implies the first three conditions. One can prove that each of the first three conditions implies the fourth one in the same way as it was done in Theorem 3.5. In view of Theorem 3.7 we have only to prove that (4) implies (5). Let $B: A \times A \rightarrow K$ be 
the bilinear map defined as follows: $B\left(e_{i}, e^{j}\right)=\delta_{i j}$ for all $i, j=1,2, \ldots, n$. We already know from the proof of Theorem 3.5 that $B$ is a surgenerate bilinear form satisfying $B(x y, z)=B(x, y z)$ for all $x, y, z \in A$, and $\phi(x)=B(1, x)$ is a Frobenius homomorphism. Therefore it is sufficient to show that $B(1, x y)=B(1, y x)$ for all $x, y \in A$. The last equality is equivalent to $B(x, y)=B(y, x)$. Recall that $z=$ $B\left(z, e^{i}\right) e_{i}=B\left(e_{i}, z\right) e^{i}$ for all $z \in A$. From (4) we see that $a e_{i} \otimes e^{i}=e_{j} \otimes e^{j} a$ for all $a \in A$. Therefore $B\left(a e_{i}, e^{j}\right) e_{j} \otimes e^{i}=e_{j} \otimes B\left(e_{i}, e^{j} a\right) e^{i}$, and so $B\left(a e_{i}, e^{j}\right)=B\left(e_{i}, e^{j} a\right)$ for all $i, j=1,2, \ldots, n, a \in A$. Thus $B(a x, y)=B(x, y a)$ for all $x, y, a \in A$. Setting $x=1$ and recalling that $B(1, y a)=B(y, a)$, we conclude that $B(a, y)=B(y, a)$ for all $a, y \in A$. The proof is complete.

\section{Invertibility of $Q$ and Azumaya Algebras}

Recall that an algebra $A$ over a commutative ring $K$ is called an Azumaya algebra if $A$ is a finitely generated projective $K$-module and the canonical homomorphism $A \otimes_{K} A^{\circ} \rightarrow \operatorname{Hom}_{K}(A, A)$ is an isomorphism of $K$-algebras, where $A^{\circ}$ is the opposite algebra to $A$. It is well-known that a $K$-algebra $A$ is Azumaya if and only if $A$ is a separable $K$-algebra which is a faithful projective finitely generated $K$-module and whose center $Z(A)$ equals $K \cdot 1$ (see [11, Theorem 5.3.24]). Let $A$ be a finitely generated $K$-module. Given any maximal ideal $m$ of $K$, one can easily check that the center $Z\left(A_{m}\right)$ of the localization $A_{m}$ of $A$ with respect to $m$ is equal to $Z(A)_{m}$. It follows from [3, Theorem 7.1] that $A$ is an Azumaya algebra over $K$ if and only if $A_{m}$ is an Azumaya algebra over $K_{m}$ for all $m$. In what follows we need the result obtained in [2, Theorem 4.1]: A $K$-algebra $A$ is Azumaya if and only if $Z(A)=1 \cdot K$ and there exist elements $a_{1}, a_{2}, \ldots, a_{m}, b_{1}, b_{2}, \ldots, b_{m} \in A$ such that $\sum_{i=1}^{m} a_{i} b_{i}=1$ and $\sum_{i=1}^{m} a_{i} x b_{i} \in Z(A)$ for all $x \in A$.

Theorem 4.1. Let $A$ be a Frobenius K-algebra with Frobenius homomorphism $\phi$ and let $e_{i}, e^{i}, i=1,2, \ldots, n$, be dual bases of $A$ with respect to $\phi$. Then the following conditions are equivalent:

(1) $Q=e_{i} \otimes e^{i} \in A \otimes_{K} A$ is invertible in $A \otimes_{K} A$;

(2) $A$ is an Azumaya K-algebra.

Proof. (1) $\Rightarrow(2)$. Let $m$ be a maximal ideal of $K, A_{m}$ a localization of the $K$ algebra $A$ with respect to $m$. We have only to prove that $A_{m}$ is Azumaya for all $m$. Clearly $A_{m}$ is a free $K_{m}$-module. Therefore without loss of generality we can assume that $A$ is a free $K$-module. In view of Theorem 3.4 (2) we can also assume that $e_{i}, e^{i}, i=1,2, \ldots, n$, are free bases of $A$. Let $R=\sum_{i=1}^{t} u_{i} \otimes v_{i}$ be the inverse element to $Q$. Since $R Q=1 \otimes 1,(1 \otimes x) R Q=1 \otimes x$ for all $x \in A$. Recalling that $(1 \otimes a) Q=Q(a \otimes 1)$ for all $a \in A$ by Theorem 3.4, we conclude that

$$
1 \otimes x=\left(\sum_{i=1}^{t} u_{i} \otimes x v_{i}\right) Q=\sum_{j=1}^{n}\left(\sum_{i=1}^{t} u_{i} e_{j} x v_{i}\right) \otimes e^{i},
$$

and so $1 \otimes x=\sum_{j=1}^{n} \psi\left(e_{j} x\right) \otimes e^{j}$, where $\psi(x)=\sum_{i=1}^{t} u_{i} x v_{i}$. Writing $x=\sum_{j=1}^{n} c_{j} e^{j}$, where $c_{j} \in K$ for all $j=1,2, \ldots, n$, we infer that $\psi\left(e_{j} x\right)=c_{j}=1 \cdot \phi\left(e_{j} x\right)$ for all $j=1,2, \ldots, n$, and hence $\psi(x)=1 \cdot \phi(x)$ for all $x \in A$. Since $A$ is a free $K$-module, $\phi\left(e_{i} e^{j}\right)=\delta_{i j}$ for all $i, j$. Therefore $\psi\left(e_{1} e^{1}\right)=1$. Setting $a_{i}=u_{i} e_{1}, b_{i}=e^{1} v_{i}$, we 
see that $\sum_{i=1}^{t} a_{i} b_{i}=\psi\left(e_{1} e^{1}\right)=1$ and

$$
\sum_{i=1}^{t} a_{i} x b_{i}=\psi\left(e_{1} x e^{1}\right)=1 \cdot \phi\left(e_{1} x e^{1}\right) \in Z(A) .
$$

By the above criteria $A$ is an Azumaya algebra over $Z(A)$. Finally, let $c \in Z(A)$. Then

$$
c=\sum_{i=1}^{t} a_{i} c b_{i}=\psi\left(e_{1} c e^{1}\right)=1 \cdot \phi\left(e_{1} c e^{1}\right) \in 1 \cdot K
$$

and so $Z(A) \subseteq 1 \cdot K$. Clearly $1 \cdot K \subseteq Z(A)$. Thus $Z(A)=K$, and $A$ is an Azumaya algebra over $K$.

$(2) \Rightarrow(1)$. It is enough to show that $Q$ is right and left invertible. We show that $Q$ is right invertible. The latter is equivalent to $Q A \otimes A=A \otimes A$. Localizing with respect to a maximal ideal of $K$, we reduce our statement to the case when $A$ is a free module over the local ring $K$. The left multiplication by $Q$ in $A \otimes A$ is an endomorphism of the free module $A \otimes A$. Therefore $Q A \otimes A=A \otimes A$ if and only if the determinant of this endomorphism is invertible. Factoring by $m A$, where $m$ is the maximal ideal of the local ring $K$, we reduce the problem to the case when $A$ is an Azumaya algebra over the field $K$. Tensoring by the algebraic closure of $K$, we may assume that $A$ is a $t \times t$ matrix algebra over $K$ for some natural number $t$. It is well-known that there exists an invertible element $g \in A$ such that $\phi(x)=\operatorname{tr}(g x)$ for all $x \in A$, where tr is the trace map in $A=M_{t}(K)$. Let $\left\{e_{i j} \mid 1 \leq i, j \leq t\right\}$ be a system of matrix units in $A$. Clearly, $g^{-1} e_{i j}, e_{j i}, 1 \leq i, j \leq t$, are dual bases of $A$ with respect to $\phi$. According to Theorem 3.4

$$
Q=\sum_{i j} g^{-1} e_{i j} \otimes e_{j i}=\left(g^{-1} \otimes 1\right) \sum_{i j} e_{i j} \otimes e_{j i},
$$

and so it is enough to show that $R=\sum_{i j} e_{i j} \otimes e_{j i}$ is invertible. One can check that $R e_{p q} \otimes e_{r s}=e_{r q} \otimes e_{p s}$ for all $1 \leq p, q, r, s \leq t$. Hence $R^{2}=1 \otimes 1$, and thus $R$ is invertible.

Theorem 4.2. Let $A$ be a Frobenius $K$-algebra with Frobenius homomorphism $\phi$, Nakayama automorphism $\alpha$ and dual bases $e_{i}, e^{i}, i=1,2, \ldots, n$. We set $u=e_{i} e^{i}$ and define the map $\Phi: A \rightarrow Z(A)$ as follows: $\Phi(x)=e^{i} x e_{i}$ for all $x \in A$. Consider the following conditions:

(1) $u$ is an invertible element of $A$;

(2) $A$ is a Frobenius $Z(A)$-algebra with Frobenius homomorphism $\Phi$;

(3) $\Phi(x)=1$ for some $x \in A$;

(4) $A$ is a separable $K$-algebra.

Then (1) $\Longrightarrow(2) \Longleftrightarrow$ (3) $\Longleftrightarrow$ (4), and (3) implies that A is a separable $K$ algebra with separability idempotent $e_{i} \otimes e_{i} x$. Furthermore, (1) implies that $A$ is a symmetric $K$-algebra.

Proof. $(1) \Longrightarrow(3)$. First of all we note that by Theorem 3.4(4) $\Phi$ is well-defined, i.e., $\Phi(x) \in Z(A)$ for all $x \in A$. Next we remark that $u$ and $\Phi$ are independent of the choice of dual bases and $y u=u \alpha(y)$ for all $y \in A$. Let $x \in A$ be the inverse element to $u$. Clearly $x y=\alpha(y) x$ for all $y \in A$, and so $1=x e_{i} e^{i}=\alpha\left(e_{i}\right) x e^{i}$. Since $\phi\left(e_{i} e^{j}\right)=\phi\left(e^{j} \alpha\left(e_{i}\right)\right)$ for all $1 \leq i, j \leq n$, we conclude that $e^{i}, \alpha\left(e_{i}\right), i=1,2, \ldots, n$, 
are dual bases of the $K$-module $A$, and hence $\Phi(x)=\alpha\left(e_{i}\right) x e^{i}=1$. Therefore (1) implies (3).

$(3) \Longrightarrow(4)$. Suppose that $\Phi(x)=1$ for some $x \in A$. We claim that $A$ is a separable $K$-algebra with separability idempotent $e=e_{i} \otimes e^{i} x$. Indeed, $\sum_{i=1}^{n}\left(e^{i} x\right) e_{i}=\Phi(x)=1$. Further by Theorem 3.4 (3) we have that (here we consider $e$ as an element of the $K$-algebra $A \otimes_{K} A^{\circ}$ with multiplication *)

$$
\begin{aligned}
e^{2} & =\left(e_{i} \otimes e^{i} x\right) *\left(e_{j} \otimes e^{j} x\right)=\sum_{i, j} e_{i} e_{j} \otimes e^{j} x e^{i} x=\sum_{j}\left(1 \otimes e^{j} x\right) \sum_{i} e_{i} e_{j} \otimes e^{i} x \\
& =\sum_{j}\left(1 \otimes e^{j} x\right) \sum_{i} e_{i} \otimes e^{j} e^{i} x=\sum_{i} e_{i} \otimes\left(\sum_{j} e^{j} x e_{j}\right) e^{i} x=e,
\end{aligned}
$$

and so $e^{2}=e$. Finally, $e *(a \otimes 1-1 \otimes a)=0$ for all $a \in A$ by Theorem 3.4 (3). Therefore $A$ is a separable $K$-algebra by [3, Proposition 1.1].

$(4) \Rightarrow(2)$. By [11, Theorem 5.3.24] $A$ is an Azumaya algebra over $Z(A)$. In particular $A$ is a projective finitely generated $Z(A)$-module. According to [11, Proposition 5.3.26] $A$ is a finite direct sum $\bigoplus_{t} A_{t}$ of Azumaya algebras $A_{t}$ of constant rank (as projective module over $Z\left(A_{t}\right)$ ). Taking into account that $\Phi$ is independent of the choice of dual bases, we may assume without loss of generality that each $e_{i}$ belongs to some $A_{t}$. Since every pair of distinct summands is orthogonal with respect to the bilinear form defined by $\phi$, we conclude that the inclusion $e_{i} \in A_{t}$ implies that $e^{i} \in A_{t}$. This observation reduces the problem to the case when $A$ is a projective module of constant rank over $Z(A)$. Making use of localization with respect to maximal ideals of $K$, we reduce the problem to the case when $K$ is a local ring with a maximal ideal $m$ and $A$ is a free $K$-module with free basis $e_{1}, e_{2}, \ldots, e_{n}$ and $\phi\left(e_{i} e^{j}\right)=\delta_{i j}$ for all $1 \leq i, j \leq n$. It follows from [2, Theorem 4.1] that $Z(A)$ is a direct summand of the $Z(A)$-module $A$ and so $Z(A)$ is a free $K$-module of finite rank. As $A$ is a separable $K$-algebra, $Z(A)$ is a separable $K$-algebra and $Z(A) / m Z(A)$ is a separable $K / m$-algebra by [3, Theorem 7.1]. Therefore $Z(A) / m Z(A)$ is a finite direct sum of fields. Recalling that $K$ is a local ring and $Z(A)$ is a free $K$-module of finite rank, we conclude that the Jacobson radical $J$ of $Z(A)$ is equal to $m Z(A)$ and $Z(A)$ is a semi-local ring, i.e., has only a finite number of maximal ideals. Making use of Nakayama's Lemma, one can easily show that a projective module of constant rank over a commutative semi-local ring is free. Therefore $A$ is a free $Z(A)$ module. Let $v_{1}, v_{2}, \ldots, v_{s}$ be a free basis of the $Z(A)$-module $A$ and $B=\left(\Phi\left(v_{i} v_{j}\right)\right)$. Clearly $\Phi$ is surgenerate if and only if $B$ is invertible in $M_{s}(Z(A))$, i.e., $\operatorname{det}(B)$ is an invertible element of $Z(A)$. The last condition is equivalent to the invertibility of $\operatorname{det}(B)+m Z(A)$ in the factor $\operatorname{ring} Z(A) / m Z(A)$. Hence we have reduced the problem to the case when $K$ is a field. Tensoring by the algebraic closure of $K$, we then reduce the problem to the case when $K$ is algebraically closed. Since $A$ is a separable $K$-algebra, it is a finite direct sum of matrix rings over $K$. Therefore without loss of generality we may assume that $A=M_{r}(K)$ for some natural number $r$. Then there exists an invertible element $y \in A$ such that $\phi(z)=\operatorname{tr}(z y)$ for all $z \in A$. Now let $\left\{e_{i j} \mid 1 \leq i, j \leq r\right\}$ be a system of matrix units. We see that $e_{i j}, e_{j i} y^{-1}, 1 \leq i, j \leq r$, are dual bases and $\Phi(z)=\sum_{i j} e_{j i} y^{-1} z e_{i j}=\operatorname{tr}\left(y^{-1} z\right) E$ for all $z \in A$, where $E$ is the identity matrix. Therefore $\Phi$ is surgenerate.

$(2) \Rightarrow(3)$. It is enough to show that if an algebra $H$ is a Frobenius algebra over its center $R$ with Frobenius homomorphism $\psi$, then $\psi(H)=R$. Localizing with 
respect to maximal ideals of $R$, we reduce the consideration to the case when $H$ is a free $R$-module. Let $h_{1}, \ldots, h_{n}$ be a basis of $H$ over $R$. Then there exists a homomorphism $f: H \rightarrow A$ of $R$-modules such that $f\left(h_{i}\right)=1$ for all $i=1,2, \ldots, n$. Since $\psi$ is a Frobenius homomorphism, there exists $h \in H$ such that $f(x)=\psi(x h)$ for all $x \in H$. In particular $1=f\left(h_{1}\right)=\psi\left(h_{1} h\right) \in \psi(H)$, and so $\psi(H)=R$.

Finally, suppose that $u$ is invertible. We set $\psi(x)=\phi(x u)$. Since $u$ is invertible, $\psi$ is a Frobenius homomorphism. Given $a, b \in A$, we have

$$
\psi(a b)=\phi(a b u)=\phi(b u \alpha(a))=\phi(b a u)=\psi(b a),
$$

because $u \alpha(a)=a u$ and so $\psi$ is symmetric. Thus $A$ is symmetric. The proof is complete.

The following result is a corollary to the proof of the above theorem.

Proposition 4.3. Let $A$ be a Frobenius algebra over $K$ with Frobenius homomorphism $\phi$ and dual bases $e_{i}, e^{i}, i=1,2, \ldots, n$. We set $u=e_{i} e^{i}$. Suppose that the ring $K$ contains the rational number field and $A$ is a separable $K$-algebra. Then $u$ is invertible.

Proof. Similarly to the proof of Theorem 4.2, we can reduce the problem to the case when $K$ is a field and $A$ is a matrix ring over $K$. Let, say, $K=M_{r}(K)$. Then it was shown in the proof of the above theorem that there exists an invertible element $y \in A$ such that $e_{i j}, e_{j i} y^{-1}, 1 \leq i, j \leq r$, are dual bases of $A$, where $\left\{e_{i j} \mid 1 \leq i, j \leq\right.$ $r\}$ is a system of matrix units. We see that $u=\sum_{i, j=1}^{r} e_{i j} e_{j i} y^{-1}=r y^{-1}$. Since $\operatorname{char}(K)=0$, we conclude that $u$ is invertible. The proof is complete.

We close our discussion with the following theorem, which follows directly from Theorem 4.1 and Theorem 3.5.

Theorem 4.4. Let $A$ be a $K$-algebra which is a free $K$-module with two bases $e_{1}, e_{2}, \ldots, e_{n}$, and let $e^{1}, e^{2}, \ldots, e^{n}$, and let $Q=e_{i} \otimes e^{i} \in A \otimes_{K} A$. Then the following conditions are equivalent:

(1) $Q^{12} Q^{13}=Q^{23} Q^{12}$ and $Q$ is invertible;

(2) $Q^{12} Q^{13}=Q^{13} Q^{23}$ and $Q$ is invertible;

(3) $Q^{23} Q^{12}=Q^{13} Q^{23}$ and $Q$ is invertible;

(4) $Q$ is invertible and $(1 \otimes a) Q=Q(a \otimes 1)$ for all $a \in A$;

(5) $A$ is an Azumaya $K$-algebra and a Frobenius algebra with Frobenius homomorphism $\phi: A \rightarrow K$ such that $\phi\left(e_{i} e^{j}\right)=\delta_{i j}$ for all $i, j=1,2, \ldots, n$.

\section{REFERENCES}

1. K. I. Beidar, Y. Fong and A. Stolin, On Frobenius Algebras and Quantum Yang-Baxter Equation, Preprint, TRITA-MAT-1994-0040, Royal Inst. of Technology, Stockholm, Sweden, 1994.

2. A. Braun, On Artin's Theorem and Azumaya Algebras, J. Algebra 77 (1982), 323-332. MR 83m:16005

3. F. De Meyer and E. Ingraham, Separable Algebras over Commutative Rings, Lecture Notes in Math. N 181, Springer-Verlag, Berlin-Heidelberg-New York, 1971. MR 43:6199

4. V. G. Drinfel'd, Hamiltonian Structures on Lie Groups, Lie Bialgebras and the Geometrical Meaning of the Classical Yang-Baxter Equation, Soviet Math. Dokl. 27 (1983), 68-71. MR 84i:58044

5. V. G. Drinfel'd, Quantum Groups, Proc. ICM-86 at Berkeley (A. Gleasson, ed.), vol. 1, AMS, 1987, pp. 798-820. MR 89f: 17017

6. S. Eilenberg and T. Nakayama, On the Dimension of Modules and Algebras, II, Nagoya Math. J. 9 (1955), 1-16. MR 17:453a 
7. L. A. Lambe and D. E. Radford, Algebraic Aspects of the Quantum Yang-Baxter Equation, J. Algebra. 154 (1992), 228-288. MR 94b:17026

8. R. G. Larson and M. E. Sweedler, An Associative Orthogonal Bilinear Form for Hopf Algebras, Amer. J. Math. 91 (1969), 75-94. MR 39:1523

9. S. Majid, Quasitriangular Hopf Algebras and Yang-Baxter Equations, International J. Modern Physics, A. 5 (1990), 1-91. MR 90k:16008

10. B. Pareigis, When Hopf Algebras are Frobenius Algebras, J. Algebra 18 (1971), 588-596. MR 43:6242

11. L. H. Rowen, Ring Theory, II, Academic Press, Inc., San Diego, 1988. MR 89h:16002

12. A. A. Stolin, On Rational Solutions of Yang-Baxter Equation for $\mathfrak{s l}(n)$, Math. Scand. 69 (1991), 59-80. MR 93d:17023

13. A. A. Stolin, On Constant Solutions of Yang-Baxter Equation for $\mathfrak{s l}(2), \mathfrak{s l}(3)$, Math. Scand. 69 (1991), 81-90. MR 93b:17053

Department of Mathematics, National Cheng-Kung University, Tainan, Taiwan

E-mail address: beidar@mail.ncku.edu.tw; fong@mail.ncku.edu.tw

Department of Mathematics, University of Göteborg, S-41296 Göteborg, Sweden

E-mail address: astolin@math.chalmers.se 